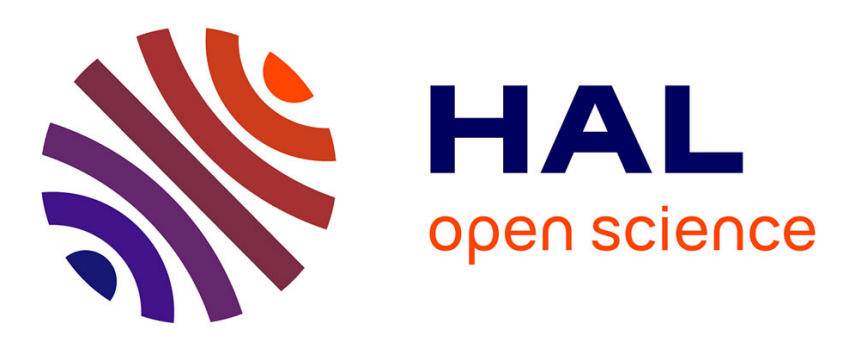

\title{
Étude START (Salivary dysfuncTion After Radioiodine Treatment) : protocole d'étude
}

Clemence Baudin, Charlotte Lussey-Lepoutre, Alice Bressand, Camille Buffet, Fabrice Menegaux, Marine Soret, David Broggio, Celine Bassinet, Christelle Huet, Catherine Ory, et al.

\section{To cite this version:}

Clemence Baudin, Charlotte Lussey-Lepoutre, Alice Bressand, Camille Buffet, Fabrice Menegaux, et al.. Étude START (Salivary dysfuncTion After Radioiodine Treatment): protocole d'étude. 37e Congrès de la Société Française d'Endocrinologie, SFE 2021, Oct 2021, LE HAVRE, France. 2021. hal-03505859

\section{HAL Id: hal-03505859 \\ https://hal.science/hal-03505859}

Submitted on 31 Dec 2021

HAL is a multi-disciplinary open access archive for the deposit and dissemination of scientific research documents, whether they are published or not. The documents may come from teaching and research institutions in France or abroad, or from public or private research centers.
L'archive ouverte pluridisciplinaire HAL, est destinée au dépôt et à la diffusion de documents scientifiques de niveau recherche, publiés ou non, émanant des établissements d'enseignement et de recherche français ou étrangers, des laboratoires publics ou privés.

$$
\text { Copyright }
$$




\section{START (SALIVARY DYSFUNCTIONS AFTER RADIOIODINE TREATMENT) :}

\section{PROTOCOLE D'ÉTUDE}

Clémence Baudin ${ }^{1}$, Charlotte Lussey-lepoutre 2,3 , Alice Bressand ${ }^{4}$, Camille Buffet ${ }^{5}$, Fabrice Menegaux ${ }^{5,6}$, Marine Soret ${ }^{2,7}$, David

Broggio $^{8}$, Céline Bassinet ${ }^{9}$, Christelle Huet ${ }^{9}$, Catherine Ory ${ }^{10}$, Laurence Leenhardt ${ }^{5}$, Gemma Armengol ${ }^{11}$, Marie-Odile Bernier ${ }^{1}$

CONTEXTE
Suite au traitement par radioiode $\left({ }^{131} \mathrm{I}\right)$ du cancer différencié de la
thyroïde, les glandes salivaires peuvent être le siège d'inflammations,
provoquant des troubles salivaires (TbS). L'incidence des TbS post-
radioiode est mal connue ainsi que les facteurs de risque cliniques et
génétiques.

\section{OBJECTIFS}

\section{MATERIELS et METHODES}

- Population : patients atteints d'un cancer différencié de la Thyroïde, candidats à un traitement par ${ }^{131}$ I (Hôpital Pitié-Salpêtrière)

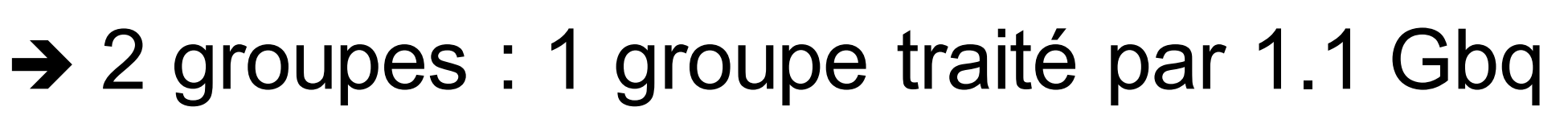

- Données collectées

$\checkmark$ Données cliniques

$\checkmark$ Questionnaires

- Troubles salivaires (E. Moreddu (2017))

- Qualité de vie (MOS SF-36)

- Sécheresse oculaire (OSDI ( )

- Nutrition (EVA des ingesta)

- Symptômes d'anxiété et dépression (échelle HAD)

\section{- Dosimétrie:}

$\checkmark 3$ dosimètres thermoluminescents (Fig. 2)

$\checkmark$ Placés à TO, enlevés 5 jours plus tard, juste avant la scintigraphie post-thérapeutique (Fig. 1)

$\checkmark$ Reconstruction dosimétrique $\rightarrow$ calcul de la dose reçue aux glandes salivaires

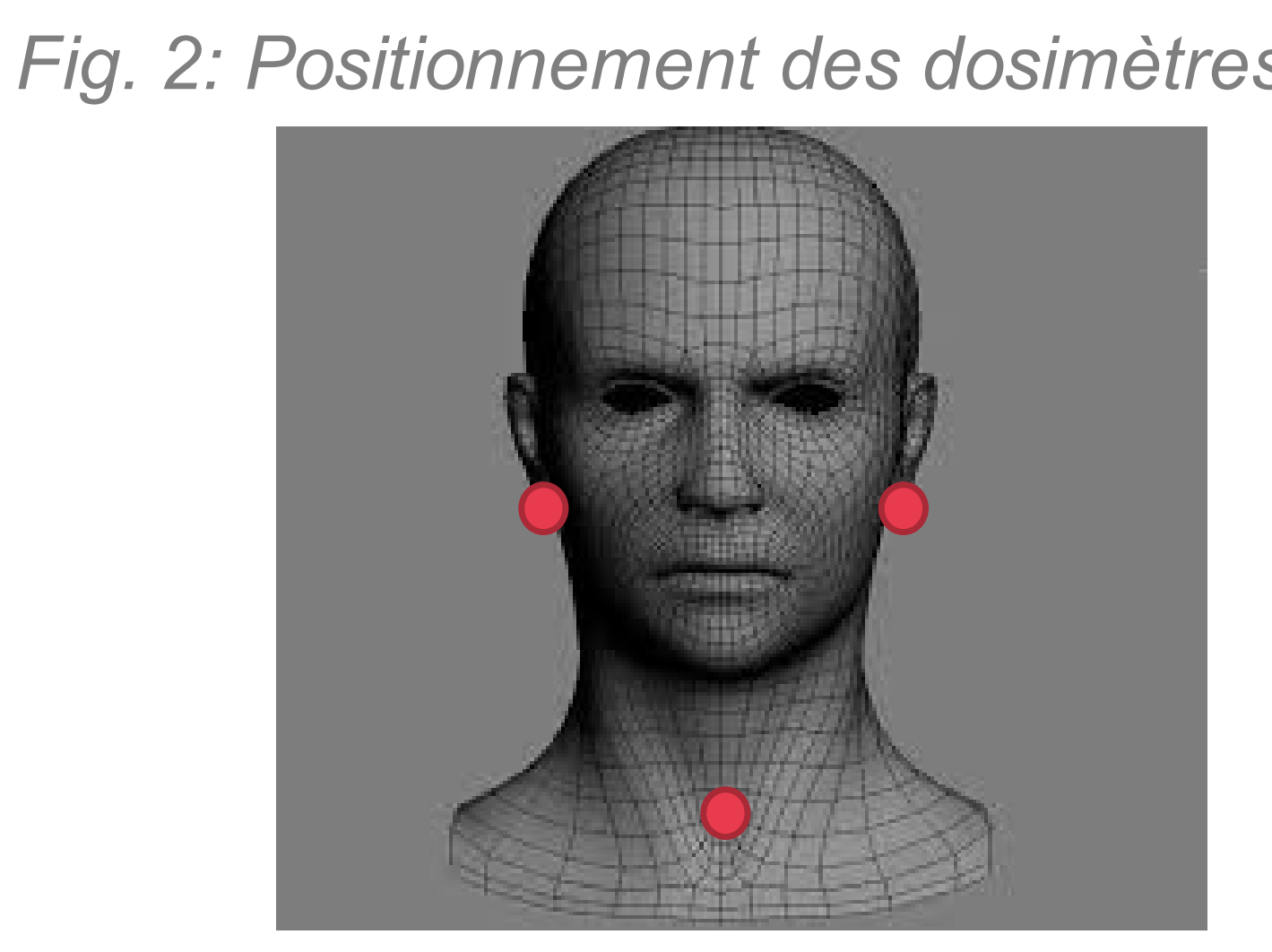

- Inclusion : le jour du traitement (T0), juste avant la prise de gélule d' ${ }^{131 \mid}$

- Suivis : à 6 (T6) et 18 (T18) mois après l'inclusion (Fig. 1)

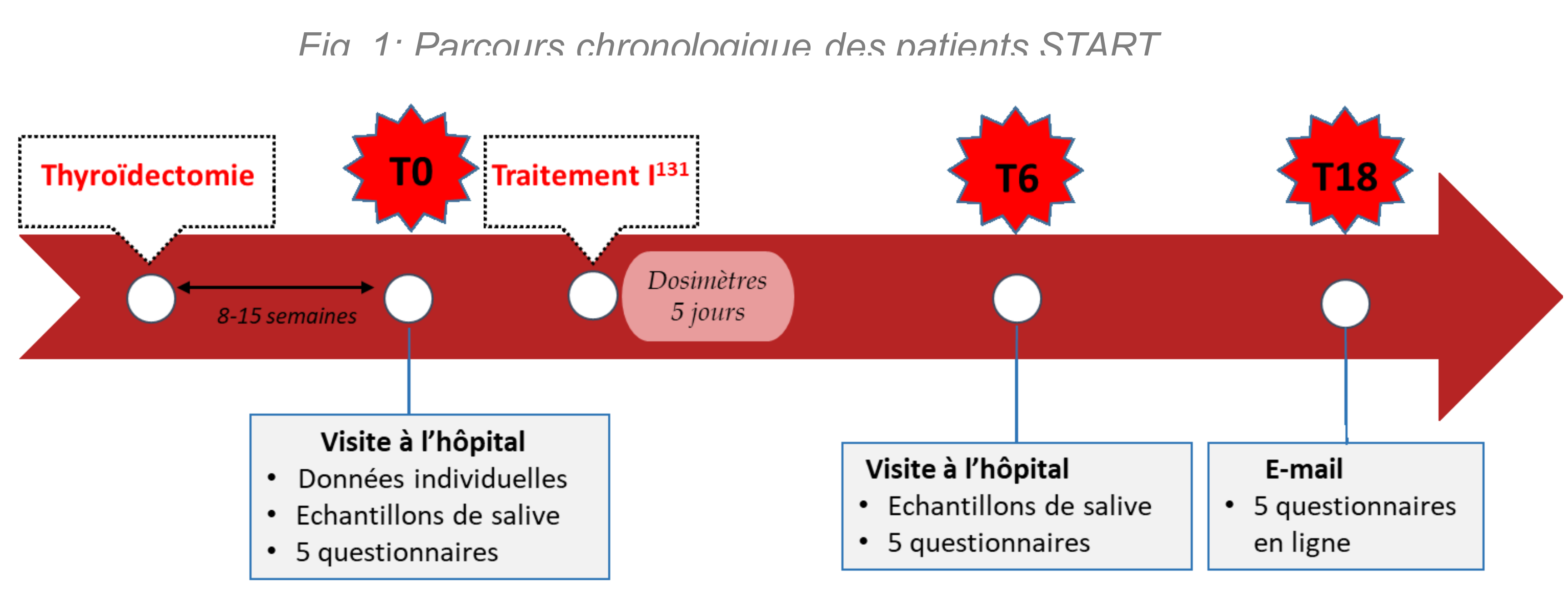

- Echantillons de salive:

$\checkmark$ Avant et après stimulation

$\checkmark$ Mesures

- Poids et volume

- Composition chimique

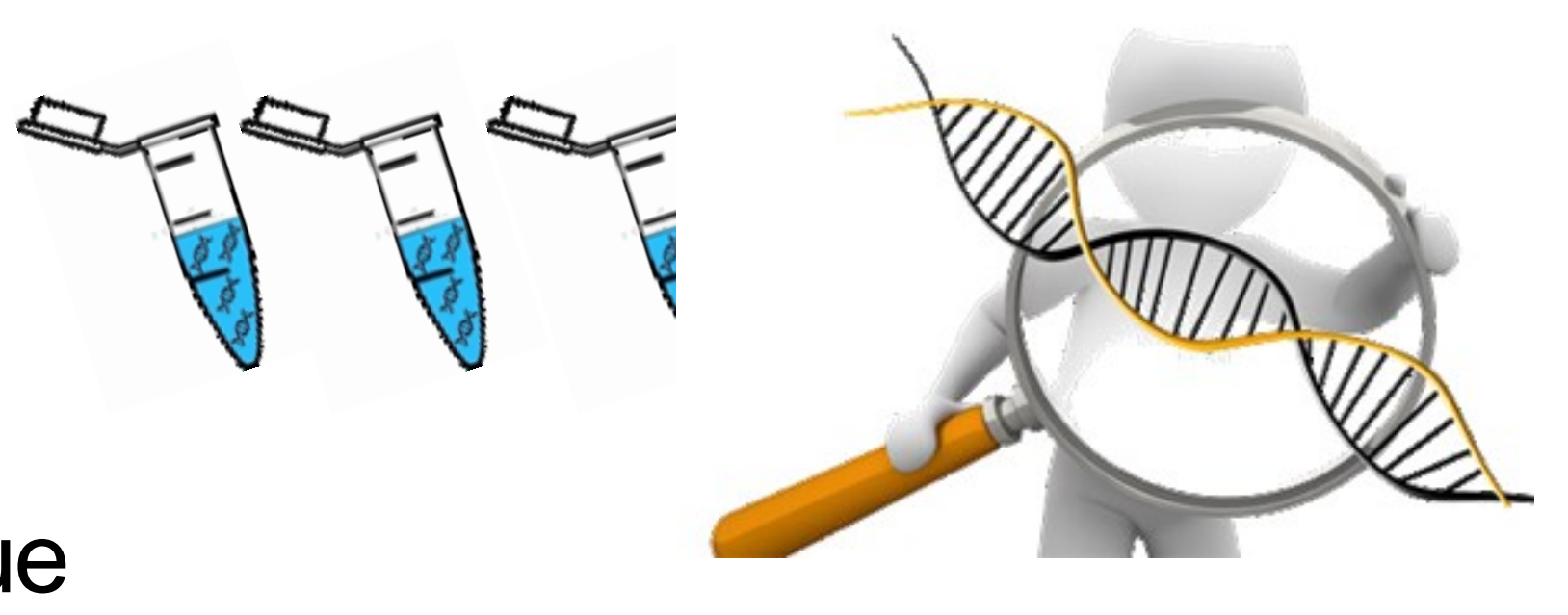

- Variants génétiques et épigénétiques

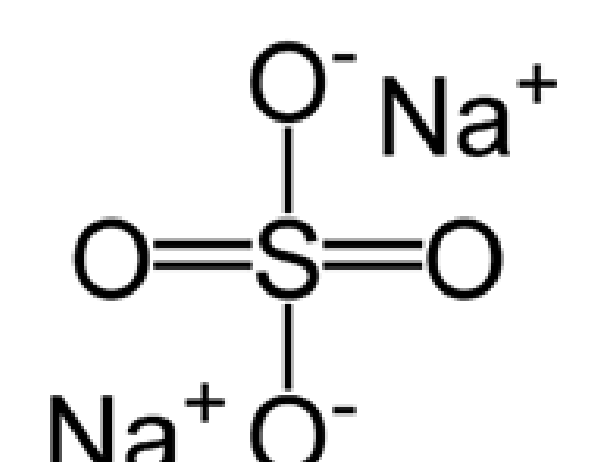

Analyses statistiques :

$\checkmark$ ACP, ACM, classifications hiérarchiques, corrélations

étude des facteurs de risques communs

$\checkmark$ Analyses appariées : comparaisons génétiques avant et après traitement par ${ }^{131}$ I

$\checkmark$ Régressions linéaires : étude des variations du flux salivaire

et des composants chimiques en fonction de la dose reçue en ${ }^{131}$ /

$\checkmark$ Régressions logistiques : estimation du risque de troubles

salivaires en fonction de la dose reçue en ${ }^{131}$ I

\section{RESULTATS}

Entre septembre 2020 et juin 2021, 139 patients $(71 \%$ de femmes et $28 \%$ d'hommes ; âge moyen= 47,1 ans $( \pm 14,1)$ ) ont été inclus. Le suivi à 6 mois a commencé en mars 2021 , et est actuellement en cours.

Cette étude permettra une évaluation de l'incidence des troubles salivaires après traitement par ${ }^{131}$, une caractérisation des patients à risque de troubles salivaires après traitement par ${ }^{131}$ I, une évaluation de la relation dose-effet entre la dose reçue par les glandes salivaires et les troubles salivaires, et une mesure de l'impact des troubles salivaires sur la qualité de vie et l'état nutritionnel à long terme.

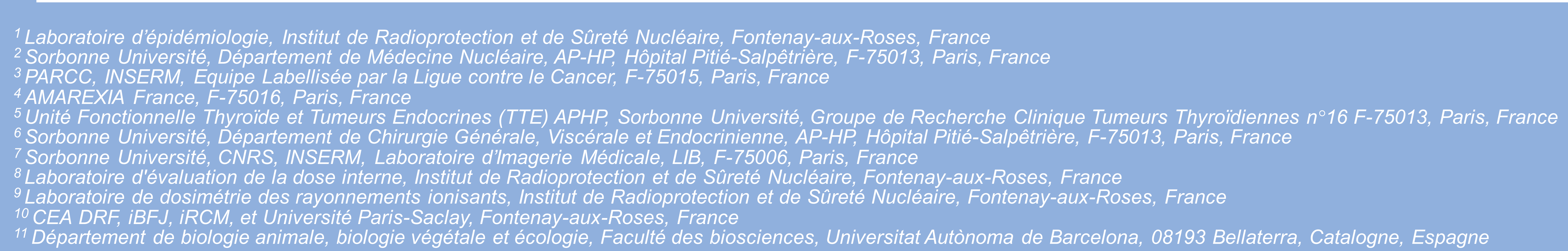

